Linz fra acciaio e arti multimediali.

La "città creativa" come laboratorio dell'utopia urbana post-industriale.

\title{
Città creativa
}

Chi conosce Linz facilmente concorda con quanti sostengono che essa sia, probabilmente, la più piccola metropoli del mondo. Può sembrare esagerato - persino paradossale - attribuire il termine "metropoli" ad una città che sfiora appena i 200.000 abitanti $^{1}$ (sono quasi 800.000 nell'area conglomerata) ma, nonostante le apparenze contrarie, Linz possiede nei suoi geni caratteriali i termini di soluzione per questo paradosso. A dispetto della sua dimensione fisica Linz è, infatti, una città globale, le cui iniziative sono calate in una realtà regionale ben precisa - quella medio-danubiana ma sono sviluppate guardando all'orizzonte sovranazionale. Questo orientamento generale contribuisce a spiegare come, nel 2009, Linz sia stata proclamata "Capitale europea della cultura" e perché, nel dicembre del 2014, sia riuscita ad entrare ufficialmente a far parte dell'UNESCO Creative Cities Network (UCCN) ${ }^{2}$ come "City of Media Arts". Non si tratta, in entrambi i casi, di episodi isolati, giacché i riconoscimenti assegnati a Linz costituiscono traguardi significativi in una lunga serie di iniziative programmate dalla città austriaca nel campo dello sviluppo sostenibile e dell'innovazione tecnologica già a partire dagli inizi degli anni '80. E', infatti, nel pieno della profonda crisi sociale che ha attraversato l'Europa in quel decennio, che Linz ha mosso i primi passi di un percorso finalizzato ad una radicale trasformazione urbana, accantonando la difficile eredità legata al suo ruolo di centro dell'industria pesante, per indirizzarsi verso un modello di città post-industriale avanzata ed ecologicamente sostenibile. Questo graduale, ma deciso cambiamento di prospettiva, si è andato sviluppando grazie ad una coerente politica di risanamento ambientale e di riqualificazione urbana, il cui compimento ha finito per modificare profondamente la vocazione culturale e lo sviluppo economico della città. Sia la trasformazione della sponda fluviale (Ars Electronica Center, Lentos, Schloßmuseum) e delle darsene industriali (Techcenter Winterhafen, Linzer Hafen), che la realizzazione di complessi interventi di

\footnotetext{
${ }^{1}$ Linz è la terza città dell'Austria dopo Vienna e Graz; con 198.181 abitanti è capoluogo dell'Oberösterreich (Alta Austria). Dei suoi abitanti, quasi il 20\% è di origine straniera; il tasso di disoccupazione resta sotto il 5\%; il reddito mensile lordo pro-capite è di $2.078 €$ (fonte: Statistik Austria, 2015).

${ }^{2}$ UCCN è stato istituito nel 2004. Organizzata in sette diversi settori sperimentali, la rete comprende attualmente circa 70 città.
} 
riqualificazione urbana (Bindermichl-Spallerhof) e di edilizia sociale (Puchenau I-II-III, SolarCity Pichling) si collocano, per alcuni aspetti, ai confini dell'utopia urbana e ci dimostrano come l'attitudine alla sperimentazione, sia alla piccola che alla grande scala, costituisca la caratteristica vitale di questa città.

L'ingresso di Linz nell'UCCN si inserisce perfettamente in questa sua genetica disponibilità alla sperimentazione, perché il network UNESCO costituisce una piattaforma di servizio fra realtà di eccellenza che hanno un comune obiettivo - collocare l'innovazione creativa come motore del sistema della crescita sostenibile e della riqualificazione urbana - che esse perseguono con strategie e strumenti diversificati fra loro. Per facilitare il processo di crescita delle città aderenti alla rete, l'UCCN stimola lo sviluppo di quelle iniziative culturali, produttive ed imprenditoriali che fanno riferimento alla cosiddetta "economia creativa" (Howkins, 2001 e 2013), teorizzata per la prima volta in ambiente anglosassone a seguito delle ricerche sviluppate sui processi innovativi di rigenerazione urbana (Hall, 1998; Landry, 2000) e sull'emergere di un nuovo, variegato strato sociale (Florida, 2000 e 2011), all'interno di una interattiva e multisensibile forma di "città creativa" (Landry-Bianchini, 1995). Rispetto al frammentato mosaico di casi studio affrontati in quest'ultimo, lungimirante saggio, che risale ormai a venti anni fa, la "creatività urbana" è diventata ormai il comun denominatore di molte politiche di sviluppo delle città contemporanee, sebbene i meccanismi di attivazione sociale ed economica, che ad esse si riferiscono, si muovano spesso lungo rotte che sono ancora difficili da analizzare e quantificare con precisione. Questa difficoltà è legata soprattutto alla sovrapposizione dei settori produttivi "creativi" con quelli tradizionali e alla complessa composizione del personale umano in essi operante, come si evince, ad esempio, dai risultati degli studi specifici (KEA, 2006 e 2015) finanziati dalla Commissione europea, nel suo crescente impegno per sostenere ed approfondire i caratteri strutturali del cosiddetto Cultural and Creative Sector (CCS). E' nel CCS, infatti, che la Commissione prevede il maggiore potenziale di crescita e di sviluppo per le città europee di media e grande dimensione, per le quali traccia prospettive di lavoro e strategie di intervento basate sui risultati conseguiti nelle politiche di riqualificazione urbana attuate in casi emblematici come quello di Linz.

Nel campo delle statistiche ufficiali, Linz può, infatti, vantare risultati convincenti, nonostante le profonde ristrutturazioni attraversate dall'industria pesante e le ricorrenti crisi economiche, sviluppatesi a cavallo del nuovo millennio, non abbiano certo risparmiato forti tensioni 
economiche e sociali al suo tessuto produttivo. Per sintetizzare il successo della riqualificazione realizzata negli ultimi decenni, basterebbe guardare alla crescita costante delle presenze e dei pernottamenti in città, che descrive una tendenza fortemente positiva soprattutto per gli ultimi due decenni.

Tab.1 - Presenze esterne e pernottamenti a Linz.

\begin{tabular}{lccccc}
\hline & 1970 & 1980 & 1990 & 2000 & 2010 \\
\hline Presenze & 146.297 & 145.086 & 204.921 & 286.125 & 408.975 \\
Pernottamenti & 258.402 & 237.413 & 351.130 & 453.554 & 693.011
\end{tabular}

Inoltre, il numero di imprese commerciali operanti nel territorio urbano è costantemente cresciuto, negli ultimi anni, con ritmi superiori alla media nazionale, nonostante gli effetti recessivi derivanti dalla crisi globale del 2007.

Tab. 2 - Imprese operanti a Linz.

\begin{tabular}{lccccc}
\hline & 2003 & 2006 & 2009 & 2012 & 2015 \\
\hline Imprese & 13.405 & 14.473 & 15.335 & 16.449 & 17.484
\end{tabular}

Oggi Linz è in grado di offrire molti più posti di lavoro ${ }^{3}$ del numero dei suoi stessi abitanti e presenta, rispetto al passato recente, una struttura produttiva molto più articolata e diversificata ${ }^{4}$, che la qualificano come uno dei più attivi distretti economici dell'Europa centrale.

Il recente riconoscimento UNESCO per la città non giunge quindi inaspettato; esso rappresenta la conseguenza delle scelte politiche e delle iniziative realizzate con continuità in questi ultimi tre decenni ${ }^{5}$. Decenni che hanno visto, più in generale, una piccola - ma dinamica - nazione come l'Austria, sostenere gli investimenti per la formazione e la ricerca tecnologica avanzata come uno dei più efficaci strumenti di crescita economica ed impegnarsi a garantire lo sviluppo compatibile delle proprie agglomerazioni urbane come volano di una equilibrata struttura produttiva e territoriale. Non sorprende quindi trovare, ormai da molti anni, Vienna al primo posto delle classifiche mondiali delle città sostenibili ${ }^{6}$ e scoprire che

\footnotetext{
${ }^{3}$ Le attività produttive della città offrono oggi ca. 205.000 posti di lavoro (fonte: Magistrat der Landeshauptstadt Linz, 2015).

${ }^{4}$ Cfr. L'analisi statistica condotta in: Österreichischer Städtebund, Statistik Austria, eds. (2013). Österreichs Städte in Zahlen 2013. Wien: Statistik Austria.

${ }^{5}$ La città ha potuto contare fino ad ora su una lunga continuità di amministrazione politica, poiché dal 1946 ad oggi si sono succeduti governi a guida SPÖ (socialdemocratici).

${ }^{6}$ Mercer's Quality of Living Rankings 2015.
} 
Graz, seconda città del paese per abitanti, figuri anch'essa come "City of Design" nel già citato network UCCN, approdatavi - proprio come Linz dopo una precedente esperienza di "Capitale europea della cultura" nel 2003. Nonostante le apparenti similitudini, il caso di Linz è tuttavia profondamente diverso da quello di Graz: se Graz, infatti, è una città storicamente sede di prestigiose istituzioni universitarie e culturali, Linz, al contrario, già agli albori della rivoluzione industriale si è configurata quasi esclusivamente come centro del lavoro operaio e della produzione manifatturiera. E' per questo motivo che riguardandola oggi, quando molti dei suoi processi di rigenerazione urbana hanno trovato una conclusione almeno parziale, la sua nuova identità di città dell'arte multimediale e dell'innovazione creativa non può che apparirci, per molti versi, ancora più interessante ed emblematica. Scopo del presente saggio è, quindi, quello di indagare i modi e le ragioni di queste profonde trasformazioni urbane svolgendo, attraverso l'analisi degli interventi realizzati negli ultimi anni, una lettura critica delle strategie di intervento sulla città.

\section{Città e acciaio}

Per descrivere efficacemente i processi di riqualificazione in atto a Linz, è necessario ripercorrere brevemente le fasi di sviluppo della sua struttura industriale, la cui fisionomia, ancora oggi, si può agevolmente riassumere in una singola parola: "acciaio". Per Linz l'acciaio è sempre stato, più che una attività economica, una parte sostanziale della propria vocazione naturale. La città sorge, infatti, sulle sponde del Danubio e ai piedi di un vasto bacino montano, da cui i minerali ferrosi sono stati estratti sin da tempi antichi. In questa area, ogni toponimo (Eisenwurzen - Eisenerz Eisenstraße) ci rimanda alla ricchezza mineraria della regione circostante. Linz è sorta sul Limes romano ma assurge solo nel medioevo al ruolo di città; viene illuminata da un momento di improvviso splendore, nel tardo '400, quando viene designata, per un breve periodo, come capitale imperiale. Operosa città di cultura protestante, già nel 1672, su un ramo addomesticato del fiume - il Fabriksarm ${ }^{7}$ - vede edificato il primo stabilimento tessile del paese - la Wollzeugfabrik - che, nel tardo '700, arriverà ad impiegare quasi 50.000 operai. La manifattura domina a lungo

\footnotetext{
${ }^{7}$ Il Fabriksarm si è formato a seguito di una piena del Danubio nel 1572, ma nei secoli successivi è andato lentamente insabbiandosi, sino a scomparire del tutto verso la fine dell'800. La forma del Fabriksarm e della adiacente Straßerinsel è ancora chiaramente leggibile nella attuale struttura urbana del Donaulände.
} 
la sponda fluviale con la sua massiccia sagoma; dismessa alla metà dell'800, viene demolita negli anni '60 per lasciare spazio alla monumentale Tabakfabrik (1929-1935) di P. Behrens ed A. Popp che, con la sua plasticità, caratterizza ancora oggi quella parte di città che viene comunemente chiamata Untere Donaulände. Fra '700 e ' 800 , favorito dalla presenza del fiume ${ }^{8}$, qui si sviluppa un rapido processo di industrializzazione che amplifica il ruolo economico della città. Linz raggiunge però la consacrazione come centro produttivo di livello internazionale solo il 13 maggio 1938, quando H. Göring inaugura ufficialmente le attività del nuovo complesso siderurgico appartenente alle Reichswerke legate al suo nome. Sulle paludose sponde orientali del Danubio viene impiantata, accanto alla imponente acciaieria, anche una nuova industria chimica'. L'acciaieria cambia per sempre il volto e il destino della città, strettamente connessi, da questo momento in poi, alle sue vicissitudini economiche e produttive. La storia della città si intreccia con quella della sua industria: Linz si trasforma, nel giro di pochi anni, da laboriosa città di provincia ad uno dei maggiori centri di produzione industriale del Reich tedesco, diventando uno dei pilastri strategici dell'economia militarizzata del nazionalsocialismo. Per Linz, sua città di adozione, Hitler predispone personalmente monumentali piani di ampliamento di cui, oggi, sopravvivono poche tracce materiali.

Con l'occupazione postbellica la città viene divisa, fra amministrazione alleata e russa, in due settori che si fronteggiano lungo il corso del Danubio: Linz resta una "città contesa" - una piccola Berlino - fino alla proclamazione dell'indipendenza nazionale nel 1955. Dopo le devastazioni belliche, nel 1946 la rinata acciaieria viene statalizzata ed assume il nome di Vereingte Österreichische Stahlindustrie (VÖEST), garantendo all'intera regione una solida ripresa economica per tutti gli anni '50-'60. La VÖEST conosce in quel periodo momenti di rapido sviluppo grazie al brevetto di un nuovo sistema di lavorazione degli acciai che porta legato nel suo, anche il nome della città: il metodo Linz-Donawitz (LD). Nel boom economico del dopoguerra, Linz consolida la sua immagine e il suo ruolo di città dell'acciaio e della chimica (Mayrhofer, 1995). I quartieri operai crescono

\footnotetext{
${ }^{8}$ La via d'acqua che passa per Linz fa parte del sistema del Rhein-Main-Donau Kanal che, realizzato lentamente fra gli anni '60-'90, permette oggi di collegare commercialmente il Mar Nero con il Mare del Nord.

${ }^{9} \mathrm{Si}$ tratta della primitiva Stickstoffwerke Ostmark AG., destinata a diventare nel dopoguerra la statalizzata Chemie Linz. Oggi, il vasto polo produttivo chimico si è strutturato nel Chemie Park, dove è concentrata la presenza di diversi gruppi internazionali operanti nel settore.
} 
velocemente e il fragile confine fra area industriale e tessuto urbano diventa sempre più incerto e problematico. Negli anni '70, la conglomerata VÖEST-ALPINE segue le sorti generali di tutta l'industria europea dell'acciaio attraversando, a causa della crisi petrolifera e di quella produttiva, una fase di progressivo declino, rafforzata, nel suo specifico caso, anche da errati investimenti finanziari. A metà degli anni '80, il licenziamento di massa di lavoratori causa conseguenze rilevanti sul tessuto economico dell'intera regione, scatenando forti tensioni sociali. Assediata dai fumi e dalle polveri della lavorazione siderurgica, la città vive avvolta in una nuvola di smog e polveri sottili, che le procurano la fama di essere la più invivibile e triste città del paese. A seguito di ripetute crisi finanziarie, il gruppo viene ristrutturato, quotato in borsa ed infine privatizzato (19931995). Le sue attività, separate in divisioni specializzate, restano oggi solo in parte attive nella produzione dell'acciaio ed abbracciano, ormai, settori differenziati che vanno dalle costruzioni meccaniche avanzate alla ricerca sui nuovi materiali. La sofisticata capacità produttiva ha permesso al gruppo di espandersi in tutto il mondo e di superare, anche se non senza difficoltà, la crisi finanziaria globale del 2007. Ancora oggi lavorano nella sola Linz, per la odierna Voestalpine, quasi 12.000 persone sulle complessive 47.000 operanti a livello mondiale ${ }^{10}$.

Il periodo più duro nei rapporti fra acciaieria e città sono stati certamente gli anni ' 80 quando, accompagnato dalle forti tensioni sociali causate dalla persistente crisi economica, l'inquinamento atmosferico raggiunge nel territorio urbano delle vette estreme, causando gravi problemi di respirazione alla popolazione (Matt, Baier, 1990). Stretta fra la consapevolezza sociale dell'importanza di lottare per ogni posto di lavoro e la ormai ineludibile necessità di una profonda riqualificazione ambientale, la città reagisce con lucidità e determinazione, inaugurando una serie di progetti di grande dimensione, con lo scopo di sperimentare modelli alternativi di insediamenti urbani eco-sostenibili. Già a partire dagli anni '60, infatti, R. Rainer ${ }^{11}$ progetta, sulla sponda sinistra del Danubio, il quartiere di Puchenau (1962-2000), mettendo alla prova un interessante schema urbano a densità media con tipologie miste. Una sorta di "città verde" (Rainer, 2000), ben inserita nel paesaggio, con una struttura di case a patio degradanti verso il fiume. Puchenau è, probabilmente, il primo tentativo nel dopoguerra di realizzare un insediamento eco-compatibile a

\footnotetext{
${ }^{10}$ Dati: Magistrat der Landeshauptstadt Linz (2013). Linz in Zahlen 2013. Linz.

${ }^{11}$ R. Rainer (1910-2004), poco noto fuori dal suo paese, è una figura di rilievo nel panorama della cultura urbanistica austriaca del dopoguerra. Architetto, realizza diversi edifici pubblici e piani urbanistici; dirige l'Ufficio urbanistico di Vienna nel periodo 1958-1963.
} 
grande scala in Europa; ad esso fa seguito, sempre per mano dello stesso architetto, un ben più ambizioso progetto per la sperimentale SolarCity ${ }^{12} \mathrm{di}$ Linz-Pichling (1992-2005), forse il più ampio e coerente modello urbano a basso consumo energetico, che sia stato realizzato compiutamente in questi ultimi decenni (Trebesburg, 2008). SolarCity è una città satellite, con un proprio centro e molte infrastrutture comunitarie, plasmata su un modello urbano che rasenta, assieme all'autarchia energetica, anche l'utopia sociale.

\section{Il Nuovo corso}

Le prime spinte verso un radicale cambiamento della politica di sviluppo urbano risalgono alla metà degli anni '70, quando una serie di iniziative artistiche frantumano la consueta immagine di Linz come "città dell'acciaio". Il passo iniziale in questa direzione viene compiuto con la costruzione, sulla sponda destra del Danubio, del Brucknerhaus (Siren K. e H., 1974) e l'istituzione del Brucknerfest, cui fanno seguito l'organizzazione del Forum Metall ${ }^{13}$ (1977), della prima Klangwolke ${ }^{14}$ per il Festival Ars Electronica (1979) ed il successivo laboratorio sperimentale del Forum Design ${ }^{15}$ (1980). Con l'eccezione dei concerti bruckneriani, ci troviamo negli altri casi di fronte a manifestazioni artistiche di massa, pensate per un pubblico internazionale e realizzate in luoghi urbani controversi o addirittura semi-abbandonati, come certamente era allora la sponda fluviale. La partecipazione dei visitatori a questi eventi si rivela

\footnotetext{
${ }^{12}$ Il quartiere modello, una vera e propria città satellite, è progettato per 5.000-6.000 abitazioni. Ha una configurazione anulare con i volumi edilizi orientati verso le insolazioni migliori, prevede un uso estensivo dell'energia solare e collegamenti pubblici elettrici.

13 Il Forum Metall - organizzato all'interno della Kunsthochschule - invade la sponda fluviale occupandola con una serie di grandi sculture di acciaio, alcune delle quali ancora oggi presenti.

${ }^{14}$ La Klangwolke (nuvola di suono) è nata da una intuizione di un gruppo di intellettuali, fra cui H. Leopoldseder (direttore della ORF, Radio di stato) e H. Gsöllpointner, rettore della allora Kunsthochscule (Accademia d'arte). La ricerca di un nuovo rapporto fra musica e tecnica mette gli organizzatori sulle tracce del lavoro del compositore bavarese W. Haupt, che proprio in quegli anni organizza, nei dintorni di Monaco, una avanguardistica "Musica per un paesaggio". Haupt viene invitato a Linz e diventa uno dei motori delle annuali Klangwolke, che ancora oggi continuano con successo in tre varianti (sperimentale, classica e per ragazzi).

${ }^{15}$ Il Forum Design - coordinato da H. Gsöllpointner, A. Hareiter, L. Ortner e P. Baum occupa un'area abbandonata, stretta fra il ponte autostradale e quello ferroviario. Il gruppo di architetti e designer Haus-Rucker-Co realizza per l'occasione una serie di padiglioni leggeri, in metallo e teloni di plastica bianca, con una forma simile a quella dei vagoni ferroviari, per sottolineare l'uso provvisorio dell'area.
} 
massiccia: la Klangwolke da sola attira sulle sponde del Danubio quasi 100.000 persone, celebrando in questa dimensione popolare l'ingresso trionfale del computer nel mondo della comunicazione di massa e della produzione artistica. Linz, stretta fra le due città capitali della musica classica, Salisburgo e Vienna, trova così nell'avanguardia artistica multimediale un inedito ruolo di guida ed un primato internazionale.

La forza innovatrice rappresentata icasticamente dalla Nike di Linz $^{16}$ e sostanziata dalla massiccia partecipazione alla Klangwolke si scontra, in quegli anni, con la desolata condizione ambientale della città, che registra a più riprese punte acute di inquinamento da smog e polveri sottili ${ }^{17}$. La reazione cittadina a questa condizione di degrado fisico, sostenuta da una ampia campagna di stampa, incoraggia la politica locale ad invertire l'ordine delle priorità strategiche ed a collocare la riqualificazione complessiva della città come obiettivo centrale delle iniziative da pianificare nel processo decisionale a tre, con Stato e Regione. Il nuovo sindaco di Linz, H. Schanovsky, dichiara programmaticamente che "Linz deve diventare la più salubre città industriale austriaca"18. A metà degli anni ' 80 , la prova di forza con le acciaierie si consuma rapidamente in una svolta eco-compatibile, favorita anche dalla, allora in atto, profonda ristrutturazione del gruppo. L'obiettivo di una drastica riduzione dell'inquinamento ambientale viene gradualmente raggiunto e, nel 2013, a 30 anni di distanza dai primi interventi, la Voestalpine può dichiarare con

\footnotetext{
${ }^{16} \mathrm{Si}$ tratta di una scultura metallica in forma della celebre statua greca, realizzata dal gruppo Haus-Rucker-Co ed issata a sbalzo sull'Hauptplatz come simbolo del Forum Metall. La sua presenza nel cornice della piazza storica provoca accese discussioni sino a quando non viene rimossa.

${ }^{17}$ Si tratta, in particolare, del "Venerdì nero" dell'11 luglio 1986, quando le soglie di sicurezza di anidride solforosa vengono superate di ben otto volte e, contemporaneamente, viene sversato cianuro nelle acque del Danubio. Una valanga di procedimenti giudiziari travolge sia la VÖEST che la Chemie Linz. La sensibilità ecologica generale è acuita dagli effetti del disastro atomico di Černobyl, verificatosi nell'aprile dello stesso anno. Anche l'inquinamento automobilistico ha una parte importante in questa situazione: Linz è ancora oggi un magnete che attrae un alto numero di pendolari che abitano nel suo circondario. Il nodo autostradale della A7, la Mühlkreisautobahn, è uno dei più trafficati del paese. L'importanza del problema automobilistico a Linz è testimoniata dal fatto che la prima forma di pianificazione per la città, nel dopoguerra, è stata la redazione di un Piano del traffico (1957), il primo del suo genere in Austria.

${ }^{18}$ Questo è il motivo centrale della sua vincente campagna politica del 1985, accompagnato dal motto "für BESSERE Luft" (per un'aria MIGLIORE) che faceva il verso all'ironia delle battute popolari sulla Smogwolke (nuvola di smog).
} 
soddisfazione il raggiungimento di tutti gli stringenti obiettivi di controllo delle immissioni nocive ${ }^{19}$.

Il risanamento atmosferico e ambientale è, come sempre in questi casi, la premessa indispensabile alla successiva opera di trasformazione urbana. Sull'onda delle decisioni prese, la città inaugura quello che è stato anche definito (Mayrhofer, Schuster, 2007) come il Neuorientierung (Nuovo corso): la riqualificazione della sponda fluviale e il risanamento delle aree dismesse diventano gli obiettivi prioritari di una più ampia strategia che punta al riposizionamento socio-economico della città nello scenario mitteleuropeo, grazie ad una politica di sostenibilità ambientale e, allo stesso tempo, di grandi progetti ed investimenti urbani sostenuti dalla mano pubblica. Grazie alla continuità della gestione politico-amministrativa della città, Linz si trasforma velocemente da "città dell'acciaio" a nodo strategico della società globale della comunicazione e della tecnologia avanzata (Mayrhofer, Schuster, Schweiger, Hermann, 2012). Sia il Brucknerhaus che le esperienze temporanee dei Forum, impegnano suoli marginali della città, focalizzandone il contrastato rapporto col fiume. Partendo da queste posizioni inedite, e talvolta persino apertamente provocatorie, la politica del Nuovo corso apre una riflessione collettiva sulle potenzialità ed il ruolo delle aree dismesse e, più in generale, sul rapporto fra città ed industria, alla ricerca di una identità urbana più sfaccettata e complessa del paesaggio turistico da cartolina rappresentato dal barocco Hauptplatz (Piazza principale). Gli interventi al di fuori del recinto storico della città, sulla disadorna sponda del fiume, costituiscono un preciso segnale della volontà di riconquistare un territorio abbandonato dagli usi collettivi. È in questo luogo fuori-centro che si materializza il Nuovo corso politico, sin dalle prime manifestazioni tecno-musicali dell'Ars Electronica, pensate come vere e proprie "azioni urbane" e realizzate per occupare il ritrovato spazio fluviale in una nuova prospettiva di "città aperta". La centralità urbana si orienta, quindi, verso il Danubio, il cui scenario naturale costituisce la cornice simbolica nella quale la città celebra se stessa e la propria rinnovata identità civile.

\section{Fuori e dentro la città: il Kulturmeile}

\footnotetext{
${ }^{19}$ Cfr. Voestalpine hat ihre Aufgabe bei der Luftsanierung erfüllt. Nachrichten.at. 5 marzo 2013.
} 
È in questo quadro generale che nasce l'idea di realizzare, per punti successivi, un asse culturale pubblico - il cosiddetto Kulturmeile ${ }^{20}$ (Miglio culturale) - che si muova lungo la sponda del Danubio e colleghi il centro storico al fiume. Una visione ambiziosa che cerca di stimolare lo sviluppo dell'intera fascia urbana danubiana fuori mura, dal Linzer Schloß (Castello) al Winterhafen (Darsena invernale), riverberando i suoi impulsi anche sulle zone peri-urbane limitrofe. La strategia dei grandi progetti, voluta fortemente dal sindaco F. Dobusch ${ }^{21}$, si avvia con la soluzione dell'annosa questione della sistemazione della Neue Galerie cittadina, arricchita della famosa collezione Gurlitt ${ }^{22}$. Sugli esiti di un concorso internazionale viene inaugurato il nuovo edificio museale del Lentos (Weber, Hofer, 2003) che si allinea sulla sponda danubiana, con i suoi 130 metri di lunghezza, collocandosi a metà strada fra il Brucknerhaus e l'Hauptplatz. Il Lentos è costruito simbolicamente attorno ad una monumentale "finestra" aperta tra città e fiume. Come già il Brucknerhaus, anche il Lentos si offre come un volume isolato e, in forza della sua chiara geometria, prova a confrontarsi dimensionalmente con le storicistiche testate ${ }^{23}$ dell'Hauptplatz verso il fiume. Le facciate vetrate del nuovo museo sono dotate di un sistema di illuminazione variabile che permette di far virare lentamente i colori delle lampade. Questo vibratile rivestimento si manifesta compiutamente di notte quando, assieme al Nibelungenbrücke (Ponte dei Nibelunghi), realizza un sistema continuo di luci colorate che, riflettendosi nell'acqua del fiume, descrivono il nuovo paesaggio urbano di Linz. Nel giro di un decennio, l'amministrazione pubblica riesce a realizzare, lungo questo asse ideale che costeggia il Danubio, una serie di importanti interventi architettonici che occupano grandi vuoti o saturano frammentarie lacune. Ma non ci si limita solo ai nuovi monumenti; quello di aver operato anche a piccola scala, costituisce infatti uno degli aspetti più interessanti del Kulturmeile di Linz, capace di aggregare, attorno ai grandi poli museali, una serie di iniziative e

\footnotetext{
${ }^{20}$ Il termine tedesco "Kulturmeile", di tradizione urbanistica ottocentesca, sta normalmente ad indicare un asse monumentale sul quale si allineano gli edifici pubblici più rappresentativi della città.

${ }^{21}$ Ricopre la carica di sindaco della città ininterrottamente dal 1988 al 2013.

${ }^{22} \mathrm{Si}$ tratta della nota collezione di W. Gurlitt, discusso collezionista e commerciante d'arte tedesco, la cui raccolta privata, messa insieme in maniera controversa negli anni del nazismo, è stata acquisita definitivamente dalla città nel 1953. È poi cronaca recente, il fortuito rinvenimento a Monaco di Baviera di centinaia di opere d'arte nella disponibilità del cugino Cornelius - figlio del più celebre Hildebrand - sulle quali si indaga ancora per chiarirne la incerta provenienza.

${ }^{23}$ Demoliti assieme al vecchio ponte ottocentesco, gli edifici di testata sono stati ricostruiti integralmente nel 1938-1940 nel quadro dei megalomani piani di Hitler per la città.
} 
di progetti di dimensione minore (Cinematograph, Atelierhaus Salzstadl, Roter Krebs IFEK, quitch, con.trust), che oggi integrano la produzione artistica di livello internazionale con interventi minuti ed eventi a misura di quartiere. La multidimensionalità delle iniziative intraprese costituisce, più in generale, uno dei motivi del successo della rigenerazione urbana realizzata lungo il fiume.

Attorno al Kulturmeile, oltre ai citati Brucknerhaus e Lentos, aprono le porte, nel giro di pochi anni, il riorganizzato museo letterario Stifterhaus (1993), la Kunstuniversität ${ }^{24}$ (1998) e lo Schoßmuseum (HoG, 2009). Sull'altra sponda del fiume, nel popolare quartiere d'oltreponte di Urfahr, si stabilisce dapprima, nel 1990, la Stadtwerkstatt ${ }^{25}$, seguita poi dal nucleo iniziale dell'Ars Electronica Center AEC (Leitner, Michl, 1998), radicalmente rinnovato ed ampliato (Treusch architecture, 2009) con la recente realizzazione dei laboratori sperimentali del Futurelab. L'attuale AEC costituisce non solo il maggiore risultato della politica culturale della città, ma rappresenta di per sé anche un convincente esempio di recupero urbano. Il progetto realizzato è riuscito, infatti, a ricollegare la sponda di Urfahr con la Hauptstrasse (Strada principale) - nonostante il forte dislivello esistente - e a ricucire le connessioni disperse con il tessuto storico del piccolo quartiere fuori mura, una volta un pittoresco borgo popolato da pescatori e uomini di fiume. L'edificio nuovo, che ha letteralmente inglobato le modeste strutture preesistenti, collega con una serie di rampe e scale i diversi livelli e offre la sua copertura centrale come una grande arena all'aperto, molto utilizzata per spettacoli ed eventi pubblici. Le sue facciate vetrate sono innervate da un sofisticato sistema di illuminazione a LED, che consente la creazione di elaborate scenografie multimediali. Con l'entrata in esercizio della AEC sulla sponda sinistra, l'asse culturale del Kulturmeile si è praticamente allargato all'intera fascia urbana attraversata dal fiume, spostando il baricentro della città ancora più decisamente all'esterno del nucleo storico. La recentissima realizzazione dello Science Park, cioè il Campus tecnologico della Johann-KeplerUniversität JKU (Caramel, 2009-2015), e della Anton Bruckner Privat Universität (Architekturbüro 1, 2008-2015) rientrano in questa strategia di rifunzionalizzazione culturale delle vaste aree disponibili che gravitano attorno al grande fiume.

\footnotetext{
${ }^{24}$ La Kunstschule der Stadt Linz è stata fondata nel 1947 e poi trasformata, nel 1973, in Hochschule, prima di diventare ufficialmente università pubblica.

${ }^{25}$ Gioco di parole fra Stadt (città) e Werkstatt (officina). La Stadtwerkstatt è un centro culturale giovanile autonomo, molto attivo e frequentato.
} 
Tornando sulla sponda destra, a partire dall'asse Lentos-Brucknerhaus non hanno tardato ad installarsi o a rinnovarsi - a seconda dei casi - una serie di infrastrutture, sia pubbliche che private, che hanno aperto una linea di connessione diretta verso le aree industriali orientali. Si tratta, in particolare, dell'ampiamento dello storico parco sportivo del Parkbad, con la nuova Eishalle (Riepl-Riepl, Kaufmann, 2008) e dell'elegante Donauforum della Oberbank (Weber, Hofer, 2010). Non sono, questi, episodi isolati; è tutto il quartiere del Donaulände che si sta configurando come distretto ri-"creativo" della città, sostenuto in questa trasformazione dalla presenza di un efficiente incubatore per imprese, il Techcenter Winterhafen ${ }^{26}$ (Gessner, 2002) e, soprattutto, dal distretto sperimentale della Tabakfabrik. La maestosa Manifattura dei tabacchi, un monumento dell'architettura industriale europea e uno dei simboli della città stessa (Bina, 2010), offre oggi una delle più convincenti declinazioni di cosa può essere un distretto creativo misto. La Kunstuniversität occupa una parte significativa degli oltre8 ha. di spazi coperti a disposizione, integrando le proprie attività con atelier esterni di design, moda, arte, comunicazione e grafica, architettura e informatica applicata. Forme comunicative ed interattive di co-working sono la regola nella configurazione degli spazi e nell'organizzazione del lavoro. La vivacità dell'ambiente produttivo e la presenza di molte imprese piccole o medio-piccole è una riprova dell'effervescenza imprenditoriale del "sistema Tabakfabrik", gestito direttamente dalla città attraverso società partecipate.

\section{Linz09}

Al culmine di queste mutazioni urbane (Frediani, 2009), la nomina a "Capitale europea della cultura" per il 2009 sancisce il ruolo internazionale ricercato dalla città per tutti gli anni ' 80 e ' $90^{27}$. Linz organizza, attraverso una società apposita, un ampio programma di iniziative affiancate da una

\footnotetext{
${ }^{26}$ Si tratta di una iniziativa economica Stato-Regione-Città, avviata già nel 1998, che supporta e finanzia start-up nel settore della tecnologia avanzata, avvalendosi anche della consulenza scientifica della JKU.

${ }^{27}$ Linz si esercita a concorrere per il ruolo di Capitale europea della cultura con una serie di iniziative preparatorie, la più importante delle quali è il "Mese della cultura europea" nel 1998. La città ottiene questo primo riconoscimento grazie ad un programma dedicato alle trasformazioni sociali e urbane nel mondo del lavoro, approfondendo le relazioni fra cultura industriale e tecnologie d'avanguardia, strutture sociali e forme artistiche. Questo successo spiana la strada alla successiva candidatura come città capitale con il motto "Linz Werkstatt der Zukunft" (Linz - laboratorio del futuro).
} 
serie di interventi concreti di riqualificazione che, avviati in anticipo di alcuni anni, vengono portati a compimento nel quadro delle celebrazioni del 2009. La filosofia che sorregge il programma di Linz09 è quella di offrire, ai visitatori come ai propri cittadini, una visione caleidoscopica e totale della città, colta dal centro storico alle periferie industriali, proiettandone al tempo stesso l'immagine sullo sfondo del panorama globalizzato nel quale essa si inserisce e opera. Per questo motivo, il padiglione riflettente $80+1$. Eine Weltreise $(80+1$. Un viaggio attorno al mondo) nell'Hauptplatz, coordinato dall'AEC, accoglie le immagini frammentate degli edifici antichi della piazza accompagnandoli con una visione virtuale e simultanea della vita quotidiana che scorre in luoghi simbolici del mondo.

L'intuizione più originale di Linz09 è stata quella di offrire nuovi punti di vista sulla città in rapida trasformazione, saggiandone, attraverso interpretazioni talvolta spiazzanti, le caratteristiche e le contraddizioni. E' stata, insomma, la città stessa ad essere terreno di ricerca e luogo di rappresentazione. Emblematico, a questo riguardo, è stato il progetto per il Pixel Hotel (any:time, 2009), una forma radicale di albergo diffuso ${ }^{28}$ che prevedeva la possibilità di soggiornare in camere ricavate in luoghi abbandonati, luoghi separati e distanti fra loro proprio come pixel isolati, da associare mentalmente per ricostituire una carta ideale della città. Linz09 ha tratteggiato una immagine inedita della quotidianità urbana, mescolando e collegando provocatoriamente cultura popolare e esperienze d'avanguardia, quartieri operai e aree industriali, autostrada e sponda fluviale. Fra gli interventi più interessanti, in questo senso, va citato Höhenrausch ${ }^{29}$ (Bow How e Riepl-Riepl, 2009), che proponeva una improbabile passeggiata sui tetti del centro culturale OK, con una serie di passerelle sospese a sbalzo sul vuoto delle strade e degli spazi pubblici sottostanti. Non solo vertigini urbane, ma anche memoria per Linz09, nel ricordo delle tragedie naziste trascritto nelle crude lacerazioni di Unter Uns (H. Steyerl) o nei testi prodotti dagli artisti di In Situ sui luoghi simbolici di un trauma storico ancora molto recente e da tutti come tale avvertito. Infine, la sfida al futuro, la riconquista dei territori marginali della città, dalla nera ed astratta Hafenhalle (Riepl-Riepl, 2009) per la darsena portuale, alla surreale ed onirica Gelbes Haus Bellevue (Fattinger, Orso e Rieper, 2009), atterrata sul

\footnotetext{
${ }^{28}$ Le camere del Pixel Hotel sono state ospitate in luoghi che andavano da una officina in disuso del centro storico alla cabina di una imbarcazione ferma alla darsena, da un negozio della periferia operaia, ad una galleria d'arte abbandonata, ai piedi del castello.

${ }^{29}$ Questa installazione è stata visitata da ca. 270.000 persone, stabilendo un nuovo primato per le mostre d'arte contemporanea nel paese.
} 
bordo della piattaforma che copre il nodo autostradale della A7, nel popolare quartiere di Bindermichl.

Non un luogo della città è stato risparmiato dal programma di interventi, e questa volontà di ricoprire l'intero mosaico di paesaggi e presenze urbane è stato forse l'aspetto più positivo di Linz09. Ma la città non è stata solo analizzata, letta e interpretata da artisti ed intellettuali, essa è stata letteralmente ricostruita e trasformata attraverso complesse architetture urbane, usando l'occasione offerta dai grandi eventi per sviluppare una politica di ampio respiro, come già accaduto in altri analoghi esempi europei (Smith, 2012). Lo Schloßmuseum ha così ricucito una lacuna dolorosa del paesaggio urbano ${ }^{30}$, ed ha allo stesso tempo offerto con la sua terrazza coperta, una veduta inedita della città dall'alto. L'inaugurazione del nuovo AEC è stato il cuore del programma, lasciando un segno incisivo e permanente sul sistema delle infrastrutture culturali della città. Anche volendo escludere dal conto il successo delle manifestazioni e delle mostre realizzate nei suoi laboratori sperimentali, basterebbe il sistema di spazi pubblici che ha rigenerato a giustificare pienamente la necessità della sua costruzione. L'equilibrato insieme di provvisorio e stabile, di eventi e infrastrutture, hanno fatto delle manifestazioni del 2009 non solo un'occasione di rilancio turistico di Linz, ma soprattutto costituito un momento nodale nella storia culturale della città. Partendo dalla memoria critica del passato, si è aperta infatti la possibilità di stilare un bilancio delle trasformazioni urbane realizzate sino a quel momento, riassunte dall'inaugurazione dello Schloßmuseum e dell'AEC. Allo stesso tempo, Linz ha cercato e potuto offrire di se stessa, l'immagine e la sostanza di una città in cambiamento, aprendo nuove prospettive di crescita verso le aree industriali orientali. È per questo motivo che il vero simbolo della nascente "città creativa" non sono stati tanto i nuovi musei, quanto invece il solitario volume del vecchio gasometro in disuso della Voestalpine, dipinto come un gigantesco tazebao per chiunque arrivi in città dall'autostrada o dalla ferrovia. Il suo cilindro metallico, tutto illuminato, segna geograficamente non solo un punto di arrivo autostradale, ma soprattutto il cuore della riqualificazione urbana che si sta aprendo verso le aree orientali, alla ricerca di un diverso equilibrio fra produzione e tutela ambientale, città e periferia.

\footnotetext{
${ }^{30} \mathrm{Si}$ tratta della ricostruzione dell'ala sud dell'antico castello, andata distrutta in seguito ad un incendio nel 1800. Da quel momento il castello, la cui presenza domina dall'alto la città e il fiume, ha perso completamente la facciata rivolta verso l'Hauptplatz, smarrendo di conseguenza anche la sua presenza urbana.
} 


\section{Città e industria}

Linz è il capoluogo di un distretto industriale molto attivo e alle sue attività produttive la città deve una buona parte della propria floridezza economica. Allo stesso tempo, la vicinanza fra aree produttive e tessuti urbani causa complessi problemi di convivenza. La riqualificazione urbana, che si è ormai consolidata sulla sponda del Danubio a partire dal Kulturmeile, si sta attualmente indirizzando verso le aree industriali ad est ${ }^{31}$ della città, non solo sfruttando settori in completa dismissione da poter riprogettare, ma anche cercando forme di possibile coesistenza fra tessuti urbani e strutture della produzione industriale. La ricerca di questa integrazione sta generando, sebbene in maniera ancora episodica, dei paesaggi ibridi, segnati da esperimenti inediti sia dal punto di vista architettonico che da quello urbano.

Uno dei primi segnali di urbanizzazione dell'area industriale orientale è stata l'istituzione del centro culturale Posthof (1984) immediatamente a margine del Linzer Hafen (porto industriale). Il successo delle sue attività ha portato al rapido radicamento del centro culturale nel frammentato tessuto dell'Hafenviertel (quartiere portuale) e ad un suo successivo ampliamento (1990). Negli anni '90, un workshop internazionale di progettazione urbana, dall'emblematico titolo "La sfida", ha prodotto una serie di progetti che hanno cercato di illustrare la possibilità di nuove relazioni fra la città e il suo vasto distretto industriale (Goldner, 1995). Come conseguenza del crescente interesse per l'espansione urbana verso est, la Voestalpine ha cominciato, di propria iniziativa, ad investire consistenti risorse in architettura contemporanea di alta qualità, sia per i propri edifici amministrativi che per le infrastrutture tecniche dell'intero polo produttivo. Negli ultimi 15 anni hanno lavorato, su brani dell'area industriale, una ampia schiera di architetti austriaci appartenenti a generazioni diverse. Questo fatto di per sé costituisce un caso raro, forse unico, nel panorama dell'industria europea contemporanea. Per trovare delle esperienze analoghe dobbiamo, infatti, raggiungere la costa occidentale degli USA e, comunque, riferirci a strutture produttive leggere, a basso tasso di inquinamento ambientale, difficilmente comparabili con le caratteristiche del colosso austriaco dell'acciaio.

\footnotetext{
${ }^{31}$ Il distretto industriale orientale segue l'ansa del Danubio per ca. $5 \mathrm{~km}$; la sua superficie è occupata da varie industrie chimiche (Chemiepark.Linz) per ca. 100 ha. e soprattutto dalla Voestalpine per ca. altri 600 ha., sino alla confluenza con la Traun, un piccolo immissario del Danubio.
} 
Ad osservare le vedute dall'alto dell'area industriale di Linz, si resta colpiti di fronte a questo paesaggio fatto di forti segni di architettura contemporanea, immersi in un magma di elementi industriali che ad essi guardano con un misto di sorpresa ed indifferenza. Questa tensione fra vecchio e nuovo, fra produttivo e urbano è alla base di una singolare contaminazione di ruoli e di funzioni. È questo il caso dello spettacolare Centro servizi per l'acciaio affacciato sul porto (x-architekten, 2004-2005), del plastico ingresso al Blocco lamiere automobilistiche (any:time, 2005), ma soprattutto del monumentale Quartier generale amministrativo della Voestalpine (Feichtinger, 2006-2009); vanno parimenti ricordati l'elegante Autosilo (x-architekten, 2007-2008) situato accanto allo spettacolare Ingresso principale (Schremmer-Jell, 2009) del distretto siderurgico, oltre al nuovo Centro visite ed esposizioni Stahlwelt (Schremmer-Jell, 2009) ed, infine, il recente Centro spirituale multiconfessionale (x-architekten, 2011). In tutti questi casi, non sorprende certo scoprire che l'acciaio, nelle sue diverse forme e consistenze materiche, costituisce il principale materiale costruttivo.

Sia il Centro servizi che il Quartier generale sono interventi edilizi di notevoli dimensioni, che entrano in diretta competizione con le strutture tecniche ed i capannoni che punteggiano l'intero comparto produttivo. In particolare, il Quartier generale, che occhieggia alla forma curvilinea della Tabakfabrik, si innalza al di sopra di una grande piastra in calcestruzzo armato che contiene parcheggi e servizi vari, ricoperti da un ampio tappeto verde. La presenza di questa area a parco è una sorpresa, per chi percorre distrattamente la Stahlstraße, collocata com'è fra gli altoforni e le tubazioni che corrono sia a terra che per aria, intrecciandosi in nodi di ogni forma e grandezza. Così come appare senza dubbio sorprendente l'aver assunto $\mathrm{i}$ complessi stilemi dell'architettura contemporanea per realizzare degli edifici, in fondo, puramente utilitaristici. Questa nuova "architettura dell'industria" non testimonia solo di una calcolata operazione di marketing aziendale (siamo moderni $=$ produciamo pulito), ma per la sua vastità e coerenza rappresenta anche un evidente tentativo di dare ordine ad un territorio che pur, a prima vista, parrebbe essere refrattario alle regole organizzative di un qualunque tessuto urbano. Nelle pieghe di questa frattura, si instaura così il conflitto irrisolto fra sistemi diversi, che è la causa e il motore di ogni trasformazione urbana.

Nel giudicare questi esperimenti non dobbiamo, tuttavia, commettere l'errore di attribuire loro le categorie ed i parametri applicati in ambiti più consueti e tradizionali. Qui l'industria, infatti, non si separa dalla città, ma ne sta cercando una contaminazione. Una sorta di lenta, ma costante 
infiltrazione che, sebbene ancora in maniera puntuale, sta tuttavia generando un nuovo paesaggio industriale misto e polifunzionale, in cui la presenza di forme di lavoro leggero e l'introduzione di elementi rappresentativi, tipici dell'architettura urbana, si fondono insieme per scandagliare i possibili modi di compenetrazione fra vita sociale e produzione industriale. Non sbaglieremmo, probabilmente, a considerare queste come le forme iniziali di quel processo che oggi tende a quella che viene comunemente descritta come l'industria del prossimo futuro, l'industria $4.0^{32}$. Quello che è certo, è che nel caso dell' acciaieria di Linz è in atto un esperimento di trasformazione che conduce ad un innalzamento della qualità urbana (e immobiliare) delle aree industriali, secondo un piano sostenuto all'interno del distretto siderurgico dalle risorse private della Voestalpine e, al suo esterno, dai piani di riqualificazione portati avanti parallelamente dalla città. Che questa, infatti, non sia da etichettare semplicemente come una illuminata iniziativa imprenditoriale, ma rappresenti davvero il segno di una profonda rigenerazione territoriale in atto, è provato dal fatto che, dopo Linz09, forse il maggior progetto urbano allo studio della locale Stadtplanung (Ufficio urbanistico) è proprio la riqualificazione delle darsene portuali ad immediato ridosso del polo industriale, sulle quali si sta sviluppando, già dal 2011, il piano operativo per il "Linzer Hafen 2024" 33. In quest'area sono previsti, infatti, forti investimenti pubblici-privati, organizzati e gestiti in una forma che si ispira esplicitamente all'esempio della HafenCity di Amburgo (Frediani, 2011). L'idea generale del piano, ancora in fase di gestazione, è quella di stimolare nell'area portuale l'insediamento di un insieme di nuove funzioni specializzate e di centri per la ricerca tecnologica applicata, assieme a forme più tradizionali di terziario $\mathrm{e}$, almeno in piccola parte, a strutture residenziali. Il piano si imposta urbanisticamente su uno schema a croce, con un ampio Kulturachse (asse culturale) trasversale che, partendo dal Posthof, attraversa il bacino mediano ed incrocia ortogonalmente l'Industrieachse (asse industriale), che si sviluppa da nord a sud e sul quale si innestano i vari impianti produttivi sia esistenti che nuovi. Al di là di

\footnotetext{
${ }^{32}$ Vedi il recente "Symposium Industrie 4.0" tenutosi nel quartiere portuale, ad ottobre 2015, su iniziativa della OÖ Wirtschftsagentur-Business Upper Austria come conferenza nazionale della piattaforma dei cluster di meccatronica.

${ }^{33}$ L'intera propietà dell'area, ca. 160 ha., è nelle mani della città. Il porto fluviale di Linz è il maggiore per estensione dell'intero bacino danubiano. Dei tre bacini esistenti, quello a nord è riservato all'ampliamento del Centro servizi della Voestalpine, quello a sud al traffico container, per quello mediano, invece, esiste un piano particolareggiato (Luger e Maul, 2013) che è risultato vincitore di una consultazione urbanistica. Complessivamente sono previsti, per la riqualificazione dell'area, investimenti per ca. 273 milioni di euro.
} 
alcune perplessità particolari (gli interramenti parziali delle darsene), non si può non concordare con l'obiettivo finale di realizzare una nuova centralità nel vecchio porto fluviale, trasformandone $i$ grandi vuoti in spazi progettati per un quartiere urbano misto, con una decisa prevalenza di attività economiche "creative". Il recupero di quest'area, oggi sottoutilizzata ed in parziale dismissione di uso, segna non solo un passo ulteriore nello sforzo di spostare il baricentro urbano verso l'area industriale, ma anche l'intenzione di sperimentare una struttura produttiva di ultima generazione, compatibile con la complessità economica e le esigenze funzionali dei nuovi tessuti urbani misti.

\section{Conclusioni}

Città industriale, città contesa, città globale, città ecologica, città creativa... Linz ha attraversato diverse fasi nella sua vita recente; tutte hanno lasciato tracce persistenti che, sovrapposte l'una alle altre, ci restituiscono il paesaggio di una città in continua e rapida evoluzione. Dalla politica abitativa a quella economica, Linz ha definito ed applicato modelli sperimentali innovativi ed ambiziosi (Mayrhofer, Schuster, 2008). Sbaglieremmo, tuttavia, a considerare le iniziative di riqualificazione, nel loro complesso, come delle azioni operate semplicemente dall'alto verso il basso. Al contrario, sin dalla metà degli anni ' 70 , è stata la mobilitazione delle istituzioni culturali pubbliche, affiancate da alcune associazioni artistiche e dalle officine sociali ad aprire il dibattito sul futuro della "città abitata" e sul suo rapporto con la "città del lavoro". Lo sforzo effettuato per risanare l'ambiente urbano e recuperare le aree industriali è il riflesso dei costanti investimenti effettuati perché la città fosse sempre più competitiva nel confronto internazionale fra i maggiori distretti culturali e produttivi dell'Europa centrale. Il successo economico delle sue attività pubbliche e private, produce oggi un alto livello di introiti comunali - di gran lunga il più alto fra le città austriache ${ }^{34}$ - provenienti in larga misura proprio dalla tassazione locale sulle attività imprenditoriali e produttive. Questo ricavo garantisce un potenziale economico costante da poter reinvestire nella trasformazione della città.

\footnotetext{
${ }^{34}$ L'introito comunale pro-capite a Linz è stato nel 2008 di 612,00 €, quasi il doppio rispetto ai $378,00 €$ di Vienna, che gode però di uno status speciale come città-regione (fonte: Magistrat der Landeshauptstadt Linz).
} 
Fra tante luci, anche alcune ombre: un grave scandalo finanziario ha recentemente travolto sia politica comunale che banche locali ${ }^{35}$, e aperto una profonda crisi di fiducia nei confronti dell'amministrazione pubblica. La crisi ha accelerato il passaggio di molti elettori sulla sponda conservatrice-populista, cosa che ha minacciato di mettere seriamente in crisi il modello di sviluppo urbano sin qui perseguito. Nonostante il quadro complessivo della città sia segnato da queste ultime, profonde crepe, Linz sembra voler continuare sul percorso già tracciato ${ }^{36}$, cercando di mettere a sistema i frammenti delle utopie urbane realizzate negli anni passati e puntando decisamente verso le possibilità offerte dalla nuova "economia creativa". Gli interventi realizzati nel distretto industriale lasciano emergere le potenzialità, sociali e produttive, di una condizione urbana caratterizzata da una fisionomia multipolare e dalla contaminazione fra produzioni ecosostenibili e tecnologie avanzate. Anche nel settore produttivo, Linz sembra quindi voler applicare il positivo effetto sinergetico fra ricerca tecnologica avanzata e sperimentazione artistica, già sperimentato con successo nel campo della creatività digitale. $\grave{E}$ in questo settore, in parte ancora inesplorato, che Linz sta collocando le sue principali risorse economiche, impostando le linee generali di un programma pensato per sostenere e sviluppare il suo ruolo nella regione danubiana per il prossimo futuro (Lechner, Philipp, 2006). Se oggi Linz ci appare, quindi, come una città stratificata e complessa ma, al tempo stesso, reattiva e dinamica, l'effetto è dovuto principalmente ai suoi rodati meccanismi di adattamento e trasformazione, emblematicamente riassunti nel motto della città, Linz verändert (Linz trasforma). Ed è vero: Linz trasforma, e si trasforma, sperimentando sul campo la sovrapposizione tra le forme della produzione di massa e quelle del lavoro "creativo", in un vasto laboratorio urbano cui

\footnotetext{
${ }^{35}$ Si tratta di uno degli scandali finanziari più consistenti che hanno segnato la cronaca austriaca negli ultimi anni. In seguito a investimenti speculativi sul corso delle valute estere (Swap), la città ha perso una cifra che varia, attualmente, fra i 500 e i 600 milioni di euro. Come raffronto, si consideri che l'ultimo bilancio consolidato (2016) di Linz ammonta a 882 milioni di euro. Per queste perdite finanziarie è attualmente in corso una complessa causa fra la città e la banca BAWAG. Occorre anche ricordare che, questo di Linz, non è stato l'unico scandalo finanziario che ha turbato il mercato austriaco; assieme agli analoghi casi di Salisburgo e Carinzia, l'emergere di forti perdite finanziarie ha rivelato la generale inadeguatezza delle amministrazioni locali a gestire complessi flussi finanziari.

${ }^{36}$ Nelle ultime elezioni regionali (27/09/2015), segnate dal problema dei profughi siriani, il partito populista FPÖ (liberali) ha scavalcato i socialdemocratici della SPÖ con una crescita di oltre 1'11\%, sebbene la guida politica del capoluogo sia ancora nelle mani della SPÖ che ha raggiunto una facile elezione del nuovo sindaco al secondo turno (11/10/2015).
} 
non sarà possibile, in futuro, fare a meno di guardare con crescente attenzione ed interesse. 
Linz fra acciaio e arti multi-mediali.

La "città creativa" come laboratorio dell'utopia urbana post-industriale.

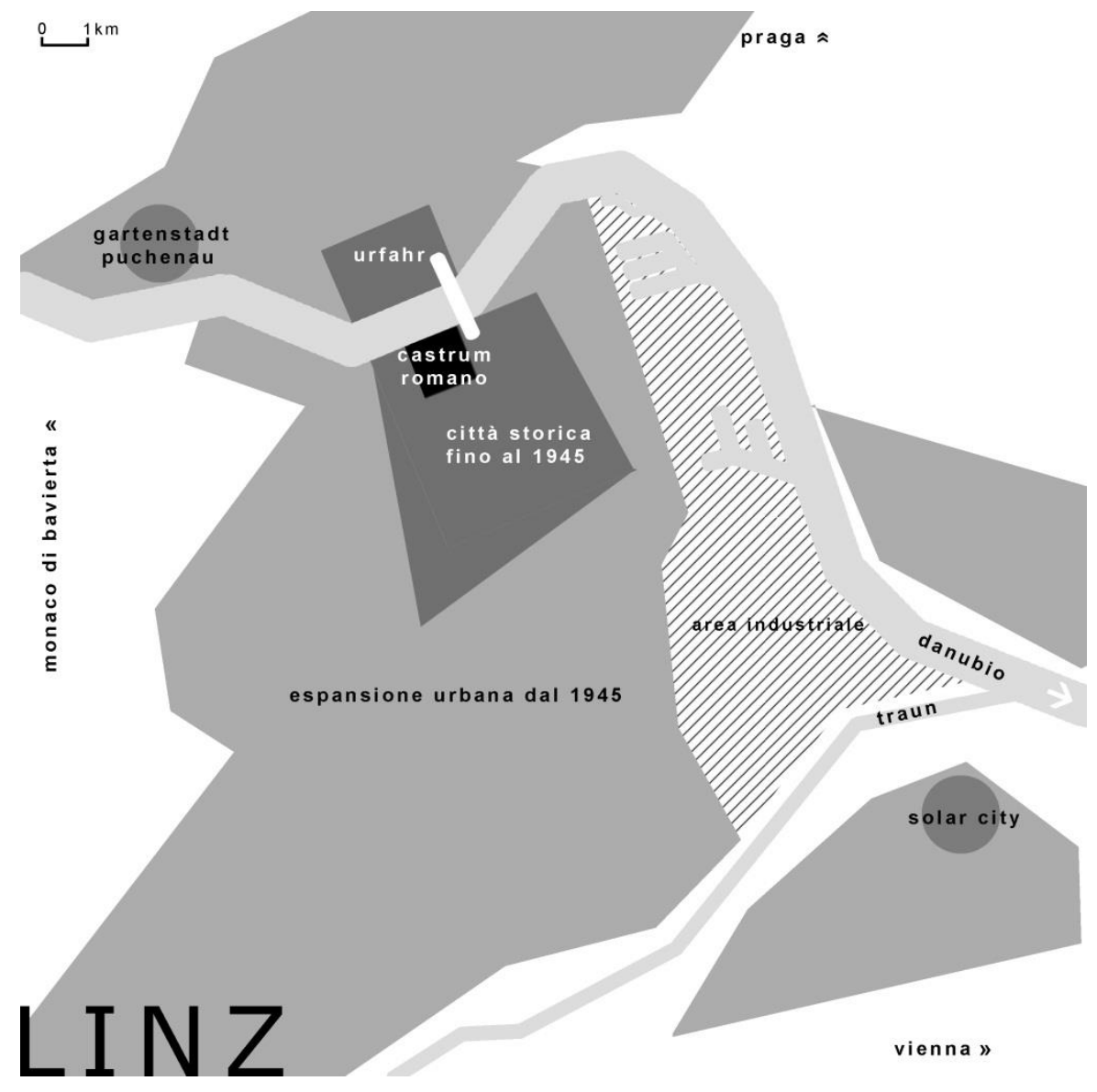

Schema urbano di Linz 


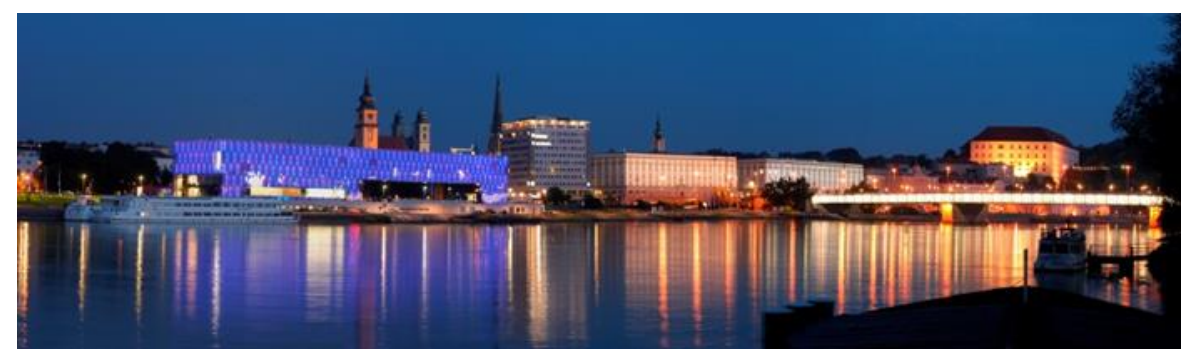

Kulturmeile: il Lentos e il ponte (foto: Stadt Linz)

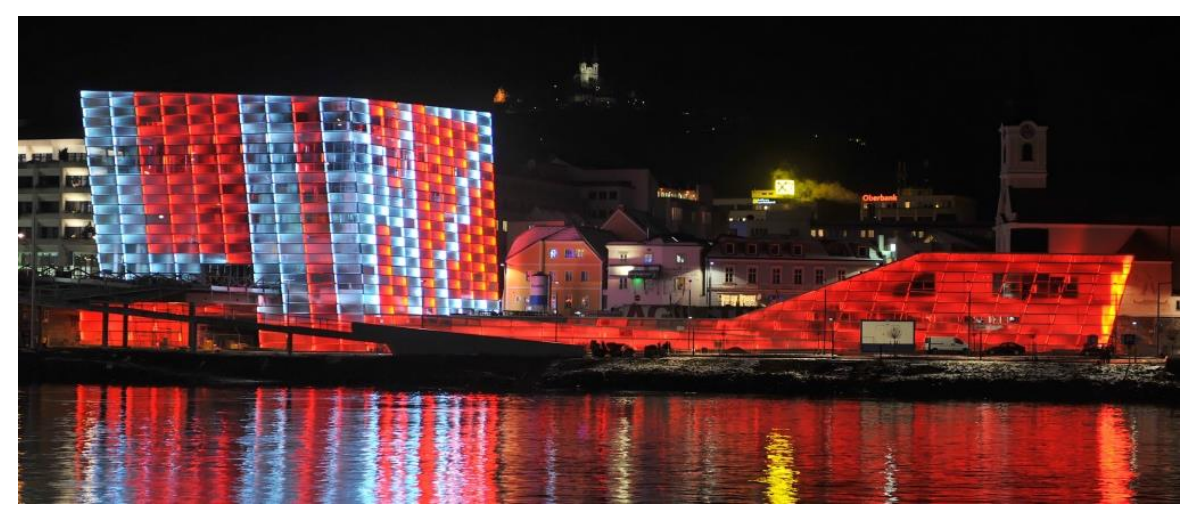

Ars Electronica Center (foto: Stadt Linz)

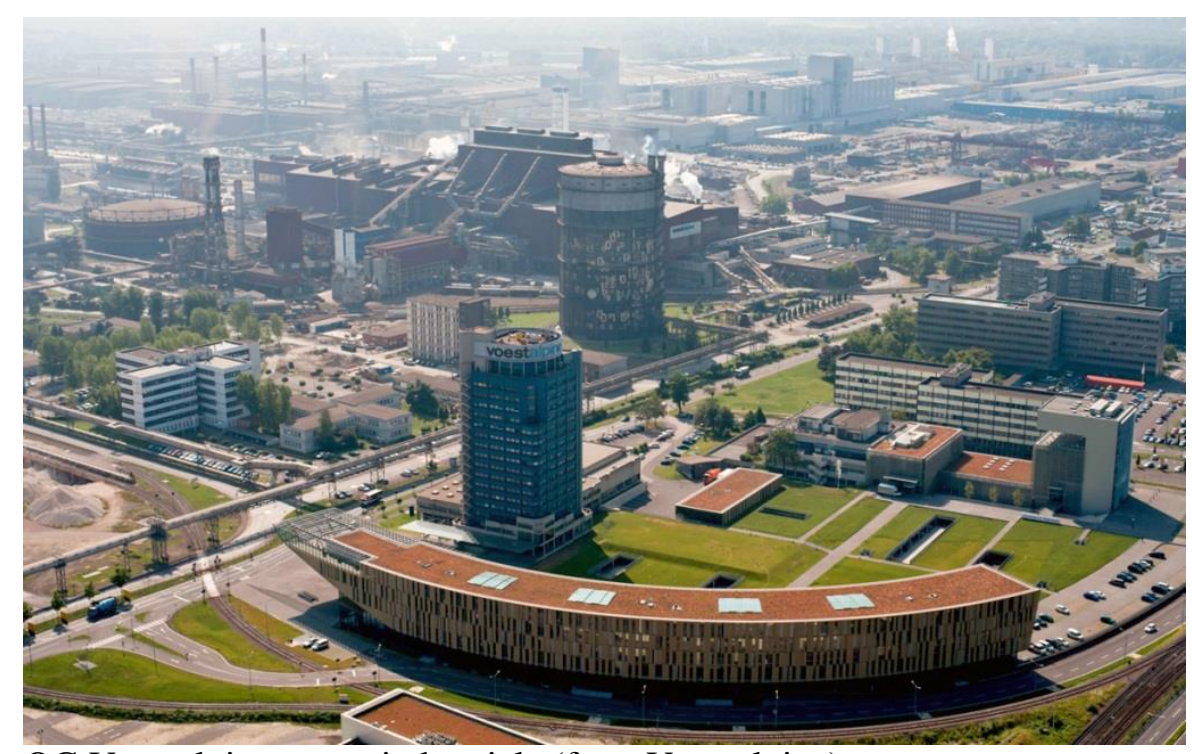

QG Voestalpine e area industriale (foto: Voestalpine) 
Durante la stesura del presente saggio è venuto improvvisamente a mancare il Dr. DI. Hans-Jörg Kaiser, responsabile del Settore Centro storico delle città di Linz (1989-1993) e di Steyr (1993-2014), per molti anni direttore del Linzer Planungsinstitut ed animatore degli Architekturtage in Steyr. L'autore lo ricorda con riconoscenza, per le indicazioni ricevute e, con amicizia, per il sodalizio culturale e umano.

Tabelle: elaborazione dell'autore su dati Magistrat der Landeshauptstadt Linz. Foto: Stadt Linz. 